\title{
Video-Based Learning and Open Online Courses
}

http://dx.doi.org/10.3991/ijet.v9i1.3354

\author{
Michail N. Giannakos ${ }^{1}$, Konstantinos Chorianopoulos ${ }^{2}$, Marco Ronchetti ${ }^{3}$, Peter Szegedi ${ }^{4}$ and Stephanie D. \\ Teasley ${ }^{5}$ \\ ${ }^{1}$ Norwegian University of Science and Technology (NTNU), Trondheim, Norway \\ ${ }^{2}$ Ionian University, Corfu, Greece \\ ${ }^{3}$ Università degli Studi di Trento, Trento, Italy \\ ${ }^{4}$ Trans-European Research \& Education Network Association (TERENA), Amsterdam, The Netherlands \\ ${ }^{5}$ University of Michigan Ann Arbor, MI, USA
}

\begin{abstract}
In this article, we provide an overview of the emerging research area on Analytics and Video-Based Learning. Although there are many and diverse ways of designing learning videos, there is limited understanding of the efficacy and usefulness of each method. Therefore, we survey the state-of-the-art in the design, development and evaluation of open and video-based courses. The main objective is to highlight the importance and benefits of analytics and to support instructors with the appropriate resources for improving the use of their courses. This can be achieved by combining and analyzing learners' interactions with other available data obtained from learners, as such video analytics open new avenues for research on open and video-based courses. In addition to the guidelines for videobased analytics we have selected five indicative case-studies that provide in-depth analysis..
\end{abstract}

Index Terms-MOOCs, Video-based learning, Review of research.

\section{INTRODUCTION AND OBJECTIVES}

Video-based learning is becoming more prominent in the world of education. Videos in education make it possible to overcome practical real-world constraints and explore the far greater possibilities provided by digital spaces. They promote student-centered learning either within the classroom or at home. Videos can also be integrated in online learning systems (LMS, portal, e-class, etc.) and can be combined with other services. For instance, learners can use in parallel video and an online chat room, forum or even video conferencing to communicate with their instructors. The combination of video with other learning services has great potential to provide to students with an integrated online learning space.

The use of video for learning has become widely employed in the past years [1]. Many universities and digital libraries have incorporated video into their instructional materials. Massive Open Online Courses (MOOCs) are becoming an increasingly important part of education. In order to support video learning, various technological tools have been developed such as Matterhorn and Centra. These tools provide an easy way for a learner who has missed a lecture to catch up, but also enable others, especially slow learners, to review difficult concepts.

Many instructors in higher education are implementing video lectures in a variety of ways, such as broadcasting lectures in real time, augmenting recordings of in-class lectures with face-to-face meetings for review purposes, and delivering lecture recordings before class to "flip the classroom" and to provide hands-on activities during class time. Other uses include showing videos that demonstrate course topics and providing supplementary video learning materials for self-study. Although there are many and diverse ways of designing learning videos, there is limited understanding of the efficacy and usefulness of each method.

\section{DESIGN AND DEVElopMent OF VideO BASED COURSES}

Developing a video-based course is a complex process that requires thorough planning and clear implementation procedure. Knowledge of learning theories and instructional implications is a pre-requisite for successful realization of the learning content with the most appropriate delivery components. There are several instructional design models and theories applied in e-learning [2, 3, 4]. However, for instructional design in the context of video courses we need to specify a simple but also effective way to incorporate this technique into practice.

In an attempt to provide explicit guidance to teachers and curriculum designers about how to develop their video courses, we will review some important key developmental principles of a course creation. Such a review may shed light into the crucial points of course development and lead to useful guidelines for the creation of an effective and attractive course.

Video is not a recent medium for teaching, but developmental principles have not widely specified. Most of the principles that apply to the traditional classroom delivery method also apply to learning from videos. However, these principles need to be extended to accommodate the rapid changes in technology and teaching practices.

\section{A. Technical and Technological Characteristics}

Video courses, as any other technological media product, must follow specific conditions regarding the quality of sound and video. Moreover, the design of the screen, the text flow and the other activities must be structured in a reasonable size and duration. Herein, there are certain thresholds regarding the sound and video quality [5]. In addition, there are rules regarding the appearance of the titles and the images of the videos $[6,7]$. Concluding, the whole combination of different media (media integration) must produce an effective and cohesive final multimedia (text, graphics, audio, video etc.) product.

\section{B. Cognitive Characteristics}

Knowledge of the target learners' cognitive characteristics is very important for developing an effective learning 
medium [8]. As such, the course creator should have in mind the following crucial questions regarding the learners' cognition:

- What knowledge and skills do they already have?

- What knowledge and skills must they acquire?

Other cognitive characteristics of learners, such as visual literacy (e.g., ability to perceive graphics), general attitudes towards technology and functional literacy (e.g., reading level) must be taken into account before the development of the video course.

\section{Personality Traits}

Learners' personality traits can facilitate or inhibit the effective use of learning strategies [9] and thus improve or deteriorate their performance. Moreover, personality traits can provide the motivational basis for using or not using particular learning strategies [10]. Knowledge about learners' characteristics regarding motivations (to learn with computer) and attitudes (towards content, technology, learning), could potentially help the course creator to develop an effective and adapted learning experience.

\section{Demographic and Cultural Characteristics}

The last but not least instructional factor to consider for the development of video-based courses is the learners' demographical and cultural characteristics. In every learning method, learners' physical characteristics play an important role in the overall success [11]. There are physical characteristics, such as fatigue, that instructor can only handle in synchronous teaching. However, several characteristics of the learner such as, visual and auditory abilities, age and sex, can provide useful information to the course creator, in order to adapt the video course into learners' capabilities.

\section{ANALytics on Video AND Open OnLINE COURSES}

Today millions of learners enjoy videos from different platforms (e.g., YouTube) on a diverse number of devices (desktop, smart phone, tablets) that create traces of billions of interactions. This amount of activity might be converted via analytics into useful information for the benefit of all video learners. As the number of learners watching videos on Web-based systems increases, more and more interactions have the potential to be gathered and analyzed. Capturing, sharing and analyzing these interactions (as datasets) can provide scholars and educators with valuable information the process of learning [12]. In addition, the combination of learner profiles with content metadata provide opportunities for adding value to learning analytics conducted on data from video-based learning activities.

Existing empirical research [1] has begun to identify the educational advantages and disadvantages of video-based learning. However, there still remain many essential unexplored aspects of video-based learning and the related challenges and opportunities; such as, how to use all the data obtained from the learner, how to combine data from different sources, and so on. One of the main challenges is to make sense of users' experiences and practices in order to redesign and optimize the settings and systems of video learning.

By taking into account learners' interactions and many other data-such as students' characteristics of gender, ethnicity, English-language skills, prior background knowledge, their success rate in each section, their emotional states, the speed at which they submit their answers, which video lectures seemed to help which students best in which sections, etc. - new avenues for research in the intersection of video-based learning and analytics are now possible.

In particular, guiding research questions and themes include:

- What might next generation of analytics enhanced video learning tools look like?

- What kind of data can be collected from open online video courses?

- How these data can help us to better understand and improve the value of video-based learning?

\section{THIS ISSUE}

The five papers selected to be part of this special issue on Video-Based Learning and Open Online Courses are briefly described below.

\section{A. Interaction with a Problem Solving Multi Video Lecture: Observing Students from Distance and Traditional Learning Courses}

The first paper describes a system prototype that allows the recording of several video streams associated with a lecture, including the instructor, projected slides, and information presented by the instructor via a computer (software or other videos, for instance). The several video streams, orchestrated using contextual and control information, are used to produce an interactive multi video object. In addition, the paper presents how a group of students interacted with a learning object captured from a problem solving lecture, and provides suggestions about how navigation facilities and visualization tools can assist us to include more contextual information during the presentation.

\section{B. EyApp \& AndrEyA-Free Apps for the Automated Recording of Lessons by Students}

This article introduces new prototype applications for automated recording of lectures using mobile devices. These applications were developed based on the experiences gained by the International Centre for Theoretical Physics (ICTP) Science Dissemination Unit (SDU) in Trieste, Italy with its open source "Enhance your Audience" (EyA) recording system. ICTP has more than 10,000 hours of automated educational recordings in the fields of physics and mathematics. Based on empirical experimentation this study shows the strong differences in video size outputs with respect to video recordings using iPads and EYA app.

\section{Developing a Framework for Creating Effective Instructional Video Podcasts}

This study proposes a comprehensive, theory-based framework for creating effective instructional video podcasts. Sixteen design characteristics were organized according to four categories and then used to develop 59 pre-calculus videos for 856 university freshmen. The results indicated that, overall, students had positive attitudes toward the use of worked-example video podcasts, noting they were useful and improved their understanding of precalculus concepts. Although more research needs to be conducted to establish a direct link between the remaining 
design features, student attitudes, and learning performance; this proposed framework provides a valuable starting point.

\section{Looking at MOOCs Rapid Growth Through the Lens of Video-Based Learning Research}

This paper provides a review of the video learning research over the last few years based on a selection of peerreviewed published academic papers. Authors propose a categorization based on the collected research papers, delineating some basic characteristics of video learning. The categorization attempts to look at MOOCs rapid growth through the lens of video-based learning research. The paper concludes by providing directions for future research related to the use of video learning.

\section{E. What Tweets Tell us About MOOC Participation}

The final paper describes the collection and analyses of the collected Twitter data output during MobiMOOC 2011. This six-week data stream includes all tweets that contain the MOOC's hashtag (\#mobiMOOC) and applies qualitative methodology to those data. The analysis examines the emotive vocabulary used to determine if there was content-sharing via tweets, and to analyze the folksonomic trends of the tweets. In addition, the paper proposes a deeper understanding of what and how MOOC participants share information using the MOOC's Twitter channel.

\section{CONCLUSIONS}

The potential of analytics for helping to make sense of learning procedures has drawn the interest of many scholars and practitioners in the last years. Analytics have demonstrated their ability to help us to understand (make sense) of many complex learning phenomena [12].

Although research on video based learning has been increased in the last years [1], a number of questions remain regarding the use and design of videos for learning [13]. In particular, little research has been conducted on the functionalities and the characteristics of educational videos. Characteristics like the quality of visuals used, cognitive load, instructors' engagement and tone of voice, as well as the pace, length, and segmentation of the video lectures need to be examined in more detail to improve the overall effectiveness of video as learning medium [14].

Given that learning videos can be extremely diverse in terms of technology, content, learning objectives etc. one of the biggest challenges [15] is to collect insights from diverse video learning experiences and systematically contribute to the development of a unified framework for the development of videos for learning.

\section{ACKNOWLEDGMENT}

We gratefully acknowledge the following reviewers. Without their assistance this special issue would not have come together:

-Christopher Brooks, University of Saskatchewan, CA

-Enrique Canessa, International Centre for Theoretical Physics - ICTP, IT

-Martin Ebner, Technische Universität Graz, AT

- Maria da Graca Campos Pimentel, Universidade de Sao Paulo, BR

—Davinia Hernández-Leo, Universitat Pompeu Fabra, ES
-Patrick Jermann, École Polytechnique Fédérale de Lausanne (EPFL), $\mathrm{CH}$

- Robin Kay, University of Ontario Institute of Technology, CA

-Apostolos Koutropoulos, UMass Boston, MA, USA

-Wolfgang Mueller, University of Education Weingarten, DE

-Evangelos Niforatos, Madeira Interactive Technologies Institute, PT

- Schulte Olaf, ETH Zürich, CH

- Mirko Raca, École Polytechnique Fédérale de Lausanne (EPFL), CH

\section{REFERENCES}

[1] M.N. Giannakos. "Exploring the video-based learning research: A review of the literature". British Journal of Educational Technology, 44(6), 2013, pp. 191-195. http://dx.doi.org/10.1111/bjet.12070

[2] W. Dick., \& L, Carey. 1990. The systematic design of instruction. New York: Harper Collins.

[3] J.E. Kemp, G.R. Morrison, and S.M. Ross. 1994. Designing effective instruction, Merrill, New York.

[4] K. Passerini, \& M. Granger. "A developmental model for distance learning using the Internet”. Computers and Education, 34(1), 2000, pp. 1-15. http://dx.doi.org/10.1016/S0360-1315(99)00024$\underline{\mathrm{X}}$

[5] J. Mullin, L. Smallwood, A. Watson, \& G. Wilson. "New techniques for assessing audio and video quality in real-time interactive communications", 2001 Retrieved on 2 Nov 2013 from http://www-mice.cs.ucl.ac.uk/multimedia/projects/etna/tutorial.pdf

[6] C. Dufour, E.G. Toms, J. Lewis, and R.M. Baecker, "User Strategies for Handling Information Tasks in Webcasts". In Proceedings of the SIGCHI Conference on Human Factors in Computing Systems CHI '05, 2005, ACM Press, New York, NY, pp. 1343-1346.

[7] J. L. Patterson, "Color/Font/Text Size: Developing Standards for Video Podcast". Proceedings of the 8th ACM SIGITE conference on Information technology education. 2007, ACM Press, New York, NY, pp. 273-274. http://dx.doi.org/10.1145/1324302. 1324362

[8] S. K. MacGregor, "Hypermedia navigation profiles: Cognitive characteristics and information processing strategies". Journal of Educational Computing Research, 1999, 20(2), pp. 189-206. http://dx.doi.org/10.2190/1MEC-C0W6-111H-YQ6A

[9] G. Blickle, "Personality traits, learning strategies, and performance". 1996, European Journal of Personality, 10, pp. 337-352. http://dx.doi.org/10.1002/(SICI)1099-0984(199612)10:5<337::A ID-PER258>3.0.CO;2-7

[10] T. Chamorro-Premuzic, A. and Furnham A. "Personality Predicts Academic Performance: Evidence from Two Longitudinal University Samples." Journal of Research in Personality, 2003, 37(4), pp.319-38. http://dx.doi.org/10.1016/S0092-6566(02)00578-0

[11] T. C. Reeves, \& J. G. Hedberg, "Interactive Learning Systems Evaluation". Educational Technology Press, 2002.

[12] G. Siemens, "Learning analytics: envisioning a research discipline and a domain of practice". In Proceedings of the 2nd International Conference on Learning Analytics and Knowledge (LAK '12), 2012, pp. 4-8. http://dx.doi.org/10.1145/2330601.2330605

[13] K. Chorianopoulos, \& M. N. Giannakos, "Usability design for video lectures", In Proc of the 11th European conference on Interactive tv and video (EuroITV'13), 2013, ACM Press, pp. 163-164.

[14] R. H. Kay. "Exploring the use of video podcasts in education: a comprehensive review of the literature". Computers in Human Behavior, 2012, 28(3), 820-831. http://dx.doi.org/10.1016/j.chb. 2012.01.011

[15] M.N. Giannakos, K. Chorianopoulos, M. Ronchetti, P. Szegedi, and S.D. Teasley, "Analytics on video-based learning" In Proceedings of the Third International Conference on Learning Analytics and Knowledge (LAK '13), ACM Press, 2013, pp. 283-284. 


\section{AUTHORS}

Michail N. Giannakos is ERCIM/Marie Curie Fellow in the Department of Computer and Information Science at Norwegian University of Science and Technology (NTNU), NO-7491, Trondheim, Norway (e-mail: michailg@idi.ntnu.no).

Konstantinos Chorianopoulos is Lecturer in the Department of Informatics at Ionian University, GR-49100, Corfu, Greece (e-mail: choko@ionio.gr).

Marco Ronchetti is a CS professor at the Department of Information Engineering and Computer Science at the University of Trento, , IT- 38050, Trento, Italy (e-mail: marco.ronchetti@unitn.it)

Peter Szegedi is Project Development Officer of Trans European Research and Education Network Association (TERENA), 1017W, Amsterdam, The Netherlands (email: szegedi@terena.org)

Stephanie D. Teasley is a research professor at the School of Information and the director of the USE Lab at the University of Michigan Ann Arbor, MI-48109-1285, Ann Arbor, USA (e-mail: steasley@umich.edu)

Submitted 10 November 2013. Published as re-submitted by the authors 11 February 2014. 